\title{
Some inequalities for the minimum eigenvalue of the Hadamard product of an $M$-matrix and its inverse
}

\section{Guanghui Cheng*, Qin Tan and Zhuande Wang}

\section{"Correspondence:}

ghcheng@uestc.edu.cn

School of Mathematical Sciences,

University of Electronic Science and

Technology of China, Chengdu,

Sichuan 611731, P.R. China

\begin{abstract}
In this paper, some new inequalities for the minimum eigenvalue of the Hadamard product of an M-matrix and its inverse are given. These inequalities are sharper than the well-known results. A simple example is shown.
\end{abstract}

AMS Subject Classification: 15A18; 15A42

Keywords: Hadamard product; M-matrix; inverse M-matrix; strictly diagonally dominant matrix; eigenvalue

\section{Introduction}

A matrix $A=\left(a_{i j}\right) \in \mathbb{R}^{n \times n}$ is called a nonnegative matrix if $a_{i j} \geq 0$. A matrix $A \in \mathbb{R}^{n \times n}$ is called a nonsingular $M$-matrix [1] if there exist $B \geq 0$ and $s>0$ such that

$$
A=s I_{n}-B \text { and } s>\rho(B),
$$

where $\rho(B)$ is a spectral radius of the nonnegative matrix $B, I_{n}$ is the $n \times n$ identity matrix. Denote by $\mathcal{M}_{n}$ the set of all $n \times n$ nonsingular $M$-matrices. The matrices in $\mathcal{M}_{n}^{-1}:=\left\{A^{-1}\right.$ : $\left.A \in \mathcal{M}_{n}\right\}$ are called inverse $M$-matrices. Let us denote

$$
\tau(A)=\min \{\operatorname{Re} \lambda: \lambda \in \sigma(A)\},
$$

and $\sigma(A)$ denotes the spectrum of $A$. It is known that [2]

$$
\tau(A)=\frac{1}{\rho\left(A^{-1}\right)}
$$

is a positive real eigenvalue of $A \in \mathcal{M}_{n}$ and the corresponding eigenvector is nonnegative. Indeed

$$
\tau(A)=s-\rho(B),
$$

if $A=s I_{n}-B$, where $s>\rho(B), B \geq 0$.

For any two $n \times n$ matrices $A=\left(a_{i j}\right)$ and $B=\left(b_{i j}\right)$, the Hadamard product of $A$ and $B$ is $A \circ B=\left(a_{i j} b_{i j}\right)$. If $A, B \in \mathcal{M}_{n}$, then $A \circ B^{-1}$ is also an $M$-matrix [3]. 
A matrix $A$ is irreducible if there does not exist a permutation matrix $P$ such that

$$
P A P^{T}=\left[\begin{array}{cc}
A_{1,1} & A_{1,2} \\
0 & A_{2,2}
\end{array}\right],
$$

where $A_{1,1}$ and $A_{2,2}$ are square matrices.

For convenience, the set $\{1,2, \ldots, n\}$ is denoted by $N$, where $n(\geq 3)$ is any positive integer. Let $A=\left(a_{i j}\right) \in \mathbb{R}^{n \times n}$ be a strictly diagonally dominant by row, denote

$$
\begin{aligned}
& R_{i}=\sum_{k \neq i}\left|a_{i k}\right|, \quad d_{i}=\frac{R_{i}}{\left|a_{i i}\right|}, \quad \forall i \in N ; \\
& s_{j i}=\frac{\left|a_{j i}\right|+\sum_{k \neq j, i}\left|a_{j k}\right| d_{k}}{\left|a_{j j}\right|}, \quad j \neq i, \forall j \in N ; \quad s_{i}=\max _{j \neq i}\left\{s_{i j}\right\}, \quad \forall i \in N ; \\
& r_{j i}=\frac{\left|a_{j i}\right|}{\left|a_{j j}\right|-\sum_{k \neq j, i}\left|a_{j k}\right|}, \quad j \neq i, \forall j \in N ; \quad r_{i}=\max _{j \neq i}\left\{r_{j i}\right\}, \quad \forall i \in N ; \\
& m_{j i}=\frac{\left|a_{j i}\right|+\sum_{k \neq j, i}\left|a_{j k}\right| r_{i}}{\left|a_{j j}\right|}, \quad j \neq i, \forall j \in N ; \quad m_{i}=\max _{j \neq i}\left\{m_{i j}\right\}, \quad \forall i \in N ; \\
& u_{j i}=\frac{\left|a_{j i}\right|+\sum_{k \neq j, i}\left|a_{j k}\right| m_{k i}}{\left|a_{j j}\right|}, \quad j \neq i, \forall j \in N ; \quad u_{i}=\max _{j \neq i}\left\{u_{i j}\right\}, \quad \forall i \in N .
\end{aligned}
$$

Recently, some lower bounds for the minimum eigenvalue of the Hadamard product of an $M$-matrix and an inverse $M$-matrix have been proposed. Let $A \in \mathcal{M}_{n}$, for example, $\tau\left(A \circ A^{-1}\right) \leq 1$ has been proven by Fiedler et al. in [4]. Subsequently, $\tau\left(A \circ A^{-1}\right)>\frac{1}{n}$ was given by Fiedler and Markham in [3], and they conjectured that $\tau\left(A \circ A^{-1}\right)>\frac{2}{n}$. Song [5], Yong [6] and Chen [7] have independently proven this conjecture. In [8], Li et al. improved the conjecture $\tau\left(A \circ A^{-1}\right) \geq \frac{2}{n}$ when $A^{-1}$ is a doubly stochastic matrix and gave the following result:

$$
\tau\left(A \circ A^{-1}\right) \geq \min _{i}\left\{\frac{a_{i i}-s_{i} R_{i}}{1+\sum_{j \neq i} s_{j i}}\right\} .
$$

In [9], Li et al. gave the following result:

$$
\tau\left(A \circ A^{-1}\right) \geq \min _{i}\left\{\frac{a_{i i}-m_{i} R_{i}}{1+\sum_{j \neq i} m_{j i}}\right\}
$$

Furthermore, if $a_{11}=a_{22}=\cdots=a_{n n}$, they have obtained

$$
\min _{i}\left\{\frac{a_{i i}-m_{i} R_{i}}{1+\sum_{j \neq i} m_{j i}}\right\} \geq \min _{i}\left\{\frac{a_{i i}-s_{i} R_{i}}{1+\sum_{j \neq i} s_{j i}}\right\}
$$

i.e., under this condition, the bound of [9] is better than the one of [8].

In this paper, our motives are to improve the lower bounds for the minimum eigenvalue $\tau\left(A \circ A^{-1}\right)$. The main ideas are based on the ones of [8] and [9]. 


\section{Some preliminaries and notations}

In this section, we give some notations and lemmas which mainly focus on some inequalities for the entries of the inverse $M$-matrix and the strictly diagonally dominant matrix.

Lemma 2.1 [6] Let $A \in \mathbb{R}^{n \times n}$ be a strictly diagonally dominant matrix by row, i.e.,

$$
\left|a_{i i}\right|>\sum_{j \neq i}\left|a_{i j}\right|, \quad \forall i \in N
$$

If $A^{-1}=\left(b_{i j}\right)$, then

$$
\left|b_{j i}\right| \leq \frac{\sum_{k \neq j}\left|a_{j k}\right|}{\left|a_{j j}\right|}\left|b_{i i}\right|, \quad j \neq i, \forall j \in N .
$$

Lemma 2.2 Let $A \in \mathbb{R}^{n \times n}$ be a strictly diagonally dominant $M$-matrix by row. If $A^{-1}=\left(b_{i j}\right)$, then

$$
b_{j i} \leq \frac{\left|a_{j i}\right|+\sum_{k \neq j, i}\left|a_{j k}\right| m_{k i}}{a_{j j}} b_{i i} \leq u_{j} b_{i i}, \quad j \neq i, \forall i \in N
$$

Proof Firstly, we consider $A \in \mathbb{R}^{n \times n}$ is a strictly diagonally dominant $M$-matrix by row. For $i \in N$, let

$$
r_{i}(\varepsilon)=\max _{j \neq i}\left\{\frac{\left|a_{j i}\right|+\varepsilon}{a_{j j}-\sum_{k \neq j, i}\left|a_{j k}\right|}\right\}
$$

and

$$
m_{j i}(\varepsilon)=\frac{r_{i}(\varepsilon)\left(\sum_{k \neq j, i}\left|a_{j k}\right|+\varepsilon\right)+\left|a_{j i}\right|}{a_{j j}}, \quad j \neq i
$$

Since $A$ is strictly diagonally dominant, then $r_{j i}<1$ and $m_{j i}<1$. Therefore, there exists $\varepsilon>0$ such that $0<r_{i}(\varepsilon)<1$ and $0<m_{j i}(\varepsilon)<1$. Let us define one positive diagonal matrix

$$
M_{i}(\varepsilon)=\operatorname{diag}\left(m_{1 i}(\varepsilon), \ldots, m_{i-1, i}(\varepsilon), 1, m_{i+1, i}(\varepsilon), \ldots, m_{n i}(\varepsilon)\right) .
$$

Similarly to the proofs of Theorem 2.1 and Theorem 2.4 in [8], we can prove that the matrix $A M_{i}(\varepsilon)$ is also a strictly diagonally dominant $M$-matrix by row for any $i \in N$. Furthermore, by Lemma 2.1, we can obtain the following result:

$$
m_{j i}^{-1}(\varepsilon) b_{j i} \leq \frac{\left|a_{j i}\right|+\sum_{k \neq j, i}\left|a_{j k}\right| m_{k i}(\varepsilon)}{m_{j i}(\varepsilon) a_{j j}} b_{i i}, \quad j \neq i, j \in N,
$$

i.e.,

$$
b_{j i} \leq \frac{\left|a_{j i}\right|+\sum_{k \neq j, i}\left|a_{j k}\right| m_{k i}(\varepsilon)}{a_{j j}} b_{i i}, \quad j \neq i, j \in N .
$$


Let $\varepsilon \longrightarrow 0^{+}$to get

$$
b_{j i} \leq \frac{\left|a_{j i}\right|+\sum_{k \neq j, i}\left|a_{j k}\right| m_{k i}}{a_{j j}} b_{i i} \leq u_{j} b_{i i}, \quad j \neq i, j \in N .
$$

This proof is completed.

Lemma 2.3 Let $A=\left(a_{i j}\right) \in \mathcal{M}_{n}$ be a strictly diagonally dominant matrix by row and $A^{-1}=$ $\left(b_{i j}\right)$, then we have

$$
\frac{1}{a_{i i}} \leq b_{i i} \leq \frac{1}{a_{i i}-\sum_{j \neq i}\left|a_{i j}\right| u_{j i}}, \quad \forall i \in N
$$

Proof Let $B=A^{-1}$. Since $A$ is an $M$-matrix, then $B \geq 0$. By $A B=B A=I_{n}$, we have

$$
1=\sum_{j=1}^{n} a_{i j} b_{j i}=a_{i i} b_{i i}-\sum_{j \neq i}\left|a_{i j}\right| b_{j i}, \quad \forall i \in N
$$

Hence

$$
1 \leq a_{i i} b_{i i}, \quad \forall i \in N
$$

or equivalently,

$$
\frac{1}{a_{i i}} \leq b_{i i}, \quad \forall i \in N
$$

Furthermore, by Lemma 2.2, we get

$$
1=a_{i i} b_{i i}-\sum_{j \neq i}\left|a_{i j}\right| b_{j i} \geq\left(a_{i i}-\sum_{j \neq i}\left|a_{i j}\right| u_{j i}\right) b_{i i}, \quad \forall i \in N,
$$

i.e.,

$$
b_{i i} \leq \frac{1}{a_{i i}-\sum_{j \neq i}\left|a_{i j}\right| u_{j i}}, \quad \forall i \in N
$$

Thus the proof is completed.

Lemma 2.4 [10] Let $A \in \mathbb{C}^{n \times n}$ and $x_{1}, x_{2}, \ldots, x_{n}$ be positive real numbers. Then all the eigenvalues of $A$ lie in the region

$$
\bigcup_{1}^{n}\left\{z \in C:\left|z-a_{i i}\right| \leq x_{i} \sum_{j \neq i} \frac{1}{x_{j}}\left|a_{j i}\right|\right\}
$$

Lemma 2.5 [11] If $A^{-1}$ is a doubly stochastic matrix, then $A e=e, A^{T} e=e$, where $e=$ $(1,1, \ldots, 1)^{T}$. 


\section{Main results}

In this section, we give two new lower bounds for $\tau\left(A \circ A^{-1}\right)$ which improve the ones in [8] and [9].

Lemma 3.1 If $A \in \mathcal{M}_{n}$ and $A^{-1}=\left(b_{i j}\right)$ is a doubly stochastic matrix, then

$$
b_{i i} \geq \frac{1}{1+\sum_{j \neq i} u_{j i}}, \quad \forall i \in N
$$

Proof This proof is similar to the ones of Lemma 3.2 in [8] and Theorem 3.2 in [9].

Theorem 3.1 Let $A \in \mathcal{M}_{n}$ and $A^{-1}=\left(b_{i j}\right)$ be a doubly stochastic matrix. Then

$$
\tau\left(A \circ A^{-1}\right) \geq \min _{i}\left\{\frac{a_{i i}-u_{i} R_{i}}{1+\sum_{j \neq i} u_{j i}}\right\} .
$$

Proof Firstly, we assume that $A$ is irreducible. By Lemma 2.5, we have

$$
a_{i i}=\sum_{j \neq i}\left|a_{i j}\right|+1=\sum_{j \neq i}\left|a_{j i}\right|+1 \quad \text { and } \quad a_{i i}>1, \quad i \in N .
$$

Denote

$$
u_{j}=\max _{i \neq j}\left\{u_{j i}\right\}=\max \left\{\frac{\left|a_{j i}\right|+\sum_{k \neq j, i}\left|a_{j k}\right| m_{k i}}{a_{j j}}\right\}, \quad j \in N .
$$

Since $A$ is an irreducible matrix, we know that $0<u_{j} \leq 1$. So, by Lemma 2.4, there exists $i_{0} \in N$ such that

$$
\left|\lambda-a_{i_{0} i_{0}} b_{i_{0} i_{0}}\right| \leq u_{i_{0}} \sum_{j \neq i_{0}} \frac{1}{u_{j}}\left|a_{j i_{0}} b_{j i_{0}}\right|
$$

or equivalently,

$$
\begin{aligned}
|\lambda| & \geq a_{i_{0} i_{0}} b_{i_{0} i_{0}}-u_{i_{0}} \sum_{j \neq i_{0}} \frac{1}{u_{j}}\left|a_{j i_{0}} b_{j i_{0}}\right| \\
& \geq a_{i_{0} i_{0}} b_{i_{0} i_{0}}-u_{i_{0}} \sum_{j \neq i_{0}} \frac{1}{u_{j}}\left|a_{j i_{0}}\right| u_{j} b_{i_{0} i_{0}} \quad \text { (by Lemma 2.2) } \\
& \geq\left(a_{i_{0} i_{0}}-u_{i_{0}} \sum_{j \neq i_{0}}\left|a_{j i_{0}}\right|\right) b_{i_{0} i_{0}} \\
& =\left(a_{i_{0} i_{0}}-u_{i_{0}} R_{i_{0}}\right) b_{i_{0} i_{0}} \\
& \geq \frac{a_{i_{0} i_{0}}-u_{i_{0}} R_{i_{0}}}{1+\sum_{j \neq i_{0}} u_{j i_{0}}} \quad \text { (by Lemma 3.1) } \\
& \geq \min _{i}\left\{\frac{a_{i i}-u_{i} R_{i}}{1+\sum_{j \neq i} u_{j i}}\right\} .
\end{aligned}
$$


Secondly, if $A$ is reducible, without loss of generality, we may assume that $A$ has the following block upper triangular form:

$$
A=\left[\begin{array}{cccc}
A_{11} & A_{12} & \cdots & A_{1 K} \\
0 & A_{22} & \cdots & A_{2 K} \\
0 & 0 & \cdots & \cdots \\
0 & 0 & 0 & A_{K K}
\end{array}\right],
$$

where $A_{i i} \in \mathcal{M}_{n_{i}}$ is an irreducible diagonal block matrix, $i=1,2, \ldots, K$. Obviously, $\tau(A \circ$ $\left.A^{-1}\right)=\min _{i} \tau\left(A_{i i} \circ A_{i i}^{-1}\right)$. Thus the reducible case is converted into the irreducible case. This proof is completed.

Theorem 3.2 If $A=\left(a_{i j}\right) \in \mathcal{M}_{n}$ is a strictly diagonally dominant by row, then

$$
\min _{i}\left\{\frac{a_{i i}-u_{i} R_{i}}{1+\sum_{j \neq i} u_{j i}}\right\} \geq \min _{i}\left\{\frac{a_{i i}-s_{i} R_{i}}{1+\sum_{j \neq i} s_{j i}}\right\}
$$

Proof Since $A$ is strictly diagonally dominant by row, for any $j \neq i$, we have

$$
\begin{aligned}
d_{j}-m_{j i} & =\frac{\left|a_{j i}\right|+\sum_{k \neq j, i}\left|a_{j k}\right|}{a_{j j}}-\frac{\left|a_{j i}\right|+\sum_{k \neq j, i}\left|a_{j k}\right| r_{i}}{a_{j j}} \\
& =\frac{\left(1-r_{i}\right) \sum_{k \neq j, i}\left|a_{j k}\right|}{a_{j j}} \\
& \geq 0,
\end{aligned}
$$

or equivalently,

$$
d_{j} \geq m_{j i}, \quad j \neq i, \forall j \in N \text {. }
$$

So, we can obtain

$$
u_{j i}=\frac{\left|a_{j i}\right|+\sum_{k \neq j, i}\left|a_{j k}\right| m_{k i}}{a_{j j}} \leq \frac{\left|a_{j i}\right|+\sum_{k \neq j, i}\left|a_{j k}\right| d_{k}}{a_{j j}}=s_{j i}, \quad j \neq i, \forall j \in N,
$$

and

$$
u_{i} \leq s_{i}, \quad \forall i \in N .
$$

Therefore, it is easy to obtain that

$$
\frac{a_{i i}-u_{i} R_{i}}{1+\sum_{j \neq i} u_{j i}} \geq \frac{a_{i i}-s_{i} R_{i}}{1+\sum_{j \neq i} s_{j i}}, \quad \forall i \in N
$$

Obviously, we have the desired result

$$
\min _{i}\left\{\frac{a_{i i}-u_{i} R_{i}}{1+\sum_{j \neq i} u_{j i}}\right\} \geq \min _{i}\left\{\frac{a_{i i}-s_{i} R_{i}}{1+\sum_{j \neq i} s_{j i}}\right\}
$$

This proof is completed. 
Theorem 3.3 If $A=\left(a_{i j}\right) \in \mathcal{M}_{n}$ is strictly diagonally dominant by row, then

$$
\min _{i}\left\{\frac{a_{i i}-u_{i} R_{i}}{1+\sum_{j \neq i} u_{j i}}\right\} \geq \min _{i}\left\{\frac{a_{i i}-m_{i} R_{i}}{1+\sum_{j \neq i} m_{j i}}\right\}
$$

Proof Since $A$ is strictly diagonally dominant by row, for any $j \neq i$, we have

$$
\begin{aligned}
r_{i}-m_{j i} & =r_{i}-\frac{\left|a_{j i}\right|+\sum_{k \neq j, i}\left|a_{j k}\right| r_{i}}{a_{j j}} \\
& =\frac{r_{i}-\left|a_{j i}\right|-\sum_{k \neq j, i}\left|a_{j k}\right|}{a_{j j}} \\
& =\frac{r_{i}\left(a_{j j}-\sum_{k \neq j, i}\left|a_{j k}\right|\right)-\left|a_{j i}\right|}{a_{j j}} \\
& =\frac{a_{j j}-\sum_{k \neq j, i}\left|a_{j k}\right|}{a_{j j}}\left(r_{i}-\frac{\left|a_{j i}\right|}{a_{j j}-\sum_{k \neq j, i}\left|a_{j k}\right|}\right) \\
& \geq 0,
\end{aligned}
$$

i.e.,

$$
r_{i} \geq m_{j i}, \quad j \neq i, \forall j \in N
$$

So, we can obtain

$$
u_{j i}=\frac{\left|a_{j i}\right|+\sum_{k \neq j, i}\left|a_{j k}\right| m_{k i}}{a_{j j}} \leq \frac{\left|a_{j i}\right|+\sum_{k \neq j, i}\left|a_{j k}\right| r_{i}}{a_{j j}}=m_{j i}, \quad j \neq i, \forall j \in N
$$

and

$$
u_{i} \leq m_{i}, \quad \forall i \in N
$$

Therefore, it is easy to obtain that

$$
\frac{a_{i i}-u_{i} R_{i}}{1+\sum_{j \neq i} u_{j i}} \geq \frac{a_{i i}-m_{i} R_{i}}{1+\sum_{j \neq i} m_{j i}}, \quad \forall i \in N
$$

Obviously, we have the desired result

$$
\min _{i}\left\{\frac{a_{i i}-u_{i} R_{i}}{1+\sum_{j \neq i} u_{j i}}\right\} \geq \min _{i}\left\{\frac{a_{i i}-m_{i} R_{i}}{1+\sum_{j \neq i} m_{j i}}\right\}
$$

Remark 3.1 According to inequalities (1) and (3), it is easy to know that

$$
b_{j i} \leq \frac{\left|a_{j i}\right|+\sum_{k \neq j, i}\left|a_{j k}\right| m_{k i}}{a_{j j}} b_{i i} \leq \frac{\left|a_{j i}\right|+\sum_{k \neq j, i}\left|a_{j k}\right| d_{k}}{a_{j j}} b_{i i}, \quad \forall i \in N .
$$

and

$$
b_{j i} \leq \frac{\left|a_{j i}\right|+\sum_{k \neq j, i}\left|a_{j k}\right| m_{k i}}{a_{j j}} b_{i i} \leq \frac{\left|a_{j i}\right|+\sum_{k \neq j, i}\left|a_{j k}\right| r_{i}}{a_{j j}} b_{i i}, \quad \forall i \in N .
$$


That is to say, the result of Lemma 2.2 is sharper than the ones of Theorem 2.1 in [8] and Lemma 2.2 in [9]. Moreover, the results of Theorem 3.2 and Theorem 3.3 are sharper than the ones of Theorem 3.1 in [8] and Theorem 3.3 in [9], respectively.

Theorem 3.4 If $A \in \mathcal{M}_{n}$ is strictly diagonally dominant by row, then

$$
\tau\left(A \circ A^{-1}\right) \geq \min _{i}\left\{1-\frac{1}{a_{i i}} \sum_{j \neq i}\left|a_{j i}\right| u_{j i}\right\} .
$$

Proof This proof is similar to the one of Theorem 3.5 in [8].

Remark 3.2 According to inequalities (2) and (4), we get

$$
1-\frac{1}{a_{i i}} \sum_{j \neq i}\left|a_{j i}\right| u_{j i} \geq 1-\frac{1}{a_{i i}} \sum_{j \neq i}\left|a_{j i}\right| s_{j i}
$$

and

$$
1-\frac{1}{a_{i i}} \sum_{j \neq i}\left|a_{j i}\right| u_{j i} \geq 1-\frac{1}{a_{i i}} \sum_{j \neq i}\left|a_{j i}\right| m_{j i} .
$$

That is to say, the bound of Theorem 3.4 is sharper than the ones of Theorem 3.5 in [8] and Theorem 3.4 in [9], respectively.

Remark 3.3 Using the above similar ideas, we can obtain similar inequalities of the strictly diagonally $M$-matrix by column.

\section{Example}

For convenience, we consider the $M$-matrix $A$ is the same as the matrix of [8]. Define the $M$-matrix $A$ as follows:

$$
A=\left[\begin{array}{cccc}
4 & -1 & -1 & -1 \\
-2 & 5 & -1 & -1 \\
0 & -2 & 4 & -1 \\
-1 & -1 & -1 & 4
\end{array}\right]
$$

1. Estimate the upper bounds for entries of $A^{-1}=\left(b_{i j}\right)$. Firstly, by Lemma 2.2(2) in [9], we have

$$
A^{-1} \leq\left[\begin{array}{cccc}
1 & 0.5833 & 0.5000 & 0.5000 \\
0.6667 & 1 & 0.5000 & 0.5000 \\
0.5000 & 0.6667 & 1 & 0.5000 \\
0.5833 & 0.5833 & 0.5000 & 1
\end{array}\right] \circ\left[\begin{array}{llll}
b_{11} & b_{22} & b_{33} & b_{44} \\
b_{11} & b_{22} & b_{33} & b_{44} \\
b_{11} & b_{22} & b_{33} & b_{44} \\
b_{11} & b_{22} & b_{33} & b_{44}
\end{array}\right] .
$$

By Lemma 2.2, we have

$$
A^{-1} \leq\left[\begin{array}{cccc}
1 & 0.5625 & 0.5000 & 0.5000 \\
0.6167 & 1 & 0.5000 & 0.5000 \\
0.4792 & 0.6458 & 1 & 0.5000 \\
0.5417 & 0.5625 & 0.5000 & 1
\end{array}\right] \circ\left[\begin{array}{llll}
b_{11} & b_{22} & b_{33} & b_{44} \\
b_{11} & b_{22} & b_{33} & b_{44} \\
b_{11} & b_{22} & b_{33} & b_{44} \\
b_{11} & b_{22} & b_{33} & b_{44}
\end{array}\right] .
$$


By Lemma 2.3 and Theorem 3.1 in [9], we get

$$
\begin{array}{ll}
0.3637 \leq b_{11} \leq 0.4430, & 0.3530 \leq b_{22} \leq 0.3870, \\
0.4000 \leq b_{33} \leq 0.4000, & 0.4000 \leq b_{44} \leq 0.4000
\end{array}
$$

By Lemma 2.3 and Lemma 3.1, we get

$$
\begin{aligned}
& 0.3791 \leq b_{11} \leq 0.4233, \quad 0.3609 \leq b_{22} \leq 0.3750, \\
& 0.4000 \leq b_{33} \leq 0.4000, \quad 0.4000 \leq b_{44} \leq 0.4000 .
\end{aligned}
$$

2. Lower bounds for $\tau\left(A \circ A^{-1}\right)$.

By Theorem 3.2 in [9], we obtain

$$
0.9755=\tau\left(A \circ A^{-1}\right) \geq 0.8000 .
$$

By Theorem 3.1, we obtain

$$
0.9755=\tau\left(A \circ A^{-1}\right) \geq 0.8250 .
$$

\section{Competing interests}

The authors declare that they have no competing interests.

\section{Authors' contributions}

All authors conceived of the study, participated in its design and coordination, drafted the manuscript, participated in the sequence alignment, and read and approved the final manuscript.

\section{Acknowledgements}

This research is supported by National Natural Science Foundations of China (No. 11101069).

Received: 31 July 2012 Accepted: 24 January 2013 Published: 21 February 2013

\section{References}

1. Berman, A, Plemmons, RJ: Nonnegative Matrices in the Mathematical Sciences. Classics in Applied Mathematics, vol. 9. SIAM, Philadelphia (1994)

2. Horn, RA, Johnson, CR: Topics in Matrix Analysis. Cambridge University Press, Cambridge (1991)

3. Fiedler, M, Markham, TL: An inequality for the Hadamard product of an M-matrix and inverse M-matrix. Linear Algebra Appl. 101, 1-8 (1988)

4. Fiedler, M, Johnson, CR, Markham, T, Neumann, M: A trace inequality for M-matrices and the symmetrizability of a real matrix by a positive diagonal matrix. Linear Algebra Appl. 71, 81-94 (1985)

5. Song, YZ: On an inequality for the Hadamard product of an M-matrix and its inverse. Linear Algebra Appl. 305, 99-105 (2000)

6. Yong, XR: Proof of a conjecture of Fiedler and Markham. Linear Algebra Appl. 320, 167-171 (2000)

7. Chen, SC: A lower bound for the minimum eigenvalue of the Hadamard product of matrix. Linear Algebra Appl. 378, 159-166 (2004)

8. Li, HB, Huang, TZ, Shen, SQ, Li, H: Lower bounds for the eigenvalue of Hadamard product of an M-matrix and its inverse. Linear Algebra Appl. 420, 235-247 (2007)

9. Li, YT, Chen, FB, Wang, DF: New lower bounds on eigenvalue of the Hadamard product of an M-matrix and its inverse. Linear Algebra Appl. 430, 1423-1431 (2009)

10. Varga, RS: Minimal Gerschgorin sets. Pac. J. Math. 15(2), 719-729 (1965)

11. Sinkhorn, R: A relationship between arbitrary positive matrices and doubly stochastic matrices. Ann. Math. Stat. 35 , 876-879 (1964)

doi:10.1186/1029-242X-2013-65

Cite this article as: Cheng et al.: Some inequalities for the minimum eigenvalue of the Hadamard product of an M-matrix and its inverse. Journal of Inequalities and Applications 2013 2013:65. 